\title{
Structural basis of fumarate hydratase deficiency
}

\author{
Sarah Picaud • Kathryn L. Kavanagh • Wyatt W. Yue • \\ Wen Hwa Lee • Susanne Muller-Knapp • \\ Opher Gileadi • James Sacchettini • Udo Oppermann
}

Received: 11 May 2009/Revised: 27 January 2011 /Accepted: 31 January 2011 /Published online: 29 March 2011

(C) The Author(s) 2011. This article is published with open access at Springerlink.com

\begin{abstract}
Fumarate hydratase catalyzes the stereospecific hydration across the olefinic double bond in fumarate leading to L-malate. The enzyme is expressed in mitochondrial and cytosolic compartments, and participates in the Krebs cycle in mitochondria, as well as in regulation of cytosolic fumarate levels. Fumarate hydratase deficiency is an autosomal recessive trait presenting as metabolic disorder with severe encephalopathy, seizures and poor neurological outcome. Heterozygous mutations are associated with a predisposition to cutaneous and uterine leiomyomas and to renal cancer. The crystal structure of human fumarate hydratase shows that mutations can be grouped into two distinct classes either affecting structural integrity of the core enzyme architecture, or are localized around the enzyme active site.
\end{abstract}

Communicated by: Johannes Zschocke

References to electronic databases: OMIM: 606812 150800, 605839, 136850; EC 4.2.1.2; Gene symbol: FH; GenBank: 19743875; URL to the interactive version of the article: http://www.ssiem.org/resources/ structures/FH/; PDB code: 3E04

Competing interest: None declared.

S. Picaud $\cdot$ K. L. Kavanagh $\cdot$ W. W. Yue $\cdot$ W. H. Lee

S. Muller-Knapp $\cdot$ O. Gileadi $\cdot$ U. Oppermann $(\square)$

Structural Genomics Consortium, University of Oxford,

Old Road Campus,

Headington OX3 7DQ, UK

e-mail: udo.oppermann@sgc.ox.ac.uk

J. Sacchettini

Department of Biochemistry \& Biophysics,

Texas A\&M University,

College Station, TX 77843-2128, USA

U. Oppermann

Botnar Research Center, NIHR Oxford Biomedical Research Unit, Oxford OX3 7LD, UK
An interactive version of this manuscript (which may contain additional mutations appended after acceptance of this manuscript) may be found on the SSIEM website at:

http://www.ssiem.org/resources/structures/FH.

Abbreviations
$\begin{array}{ll}\text { FH } & \text { Fumarate hydratase } \\ \text { FHD } & \text { Fumarate hydratase deficiency } \\ \text { MCUL1 } & \text { Multiple cutaneous and uterine leiomyomata } \\ \text { HLRC } & \begin{array}{l}\text { Hereditary leiomyomatosis and renal cancer } \\ \text { syndrome }\end{array}\end{array}$

\section{Introduction}

Fumarate hydratase $(\mathrm{FH})$ and succinate dehydrogenase are two integral enzyme components of the Krebs cycle, and besides their essential role in the TCA cycle, can act as tumour suppressors (King et al. 2006). The FH gene codes for fumarate hydratase (or fumarase; EC 4.2.1.2), which catalyzes the stereospecific, reversible hydratation of fumarate to $L$-malate. The $F H$ gene localized at 1q42.1 codes for differentially processed, but sequence-wise identical cytosolic and mitochondrial forms. Whereas the mitochondrial enzyme is part of the TCA cycle, the cytosolic form is thought to utilize fumarate derived from different sources. Deficiency in FH activity causes an impaired energy production by interrupting the flow of metabolites through the Krebs cycle. Accumulation of fumarate is thought to competitively inhibit 2-oxo-glutarate dependent dioxygenases that regulate hypoxia inducible factor (HIF), thus activating oncogenic hypoxia pathways (Ratcliffe, 2007).

Due to their essential role in energy production, enzyme deficiencies result in early onset of severe encephalopathy (Kerrigan et al. 2000). Accordingly, autosomal recessive fumarate hydratase deficiency (FHD) caused by mutations in the $\mathrm{FH}$ gene results in fumaric aciduria, and common clinical 
Table 1 X-ray data collection and refinement statistics

\begin{tabular}{ll}
\hline Data collection & \\
\hline Space group & $\mathrm{P}{ }_{2} 21$ \\
$\mathrm{a}, \mathrm{b}, \mathrm{c}(\AA)$ & $188.5,188.5,114.6$ \\
$\gamma$ & $120^{\circ}$ \\
Wavelength $(\AA)$ & 1.000 \\
Resolution $(\AA)^{*}$ & $25.0-1.95(2.06-1.95)$ \\
$\mathrm{R}_{\text {merge }}(\%)^{*}$ & $0.141(0.732)$ \\
$\mathrm{I} / \mathrm{I}^{*}$ & $9.7(2.0)$ \\
Completeness $(\%)^{*}$ & $99.3(96.8)$ \\
Redundancy & \\
Refinement & $6.2(5.0)$ \\
Resolution $(\AA)$ & \\
No. reflections & $41.27-1.90$ \\
$\mathrm{R}_{\text {work }} / \mathrm{R}_{\text {free }}(\%)$ & 168629 \\
No. atoms & $19.7 / 24.4$ \\
Protein & \\
Ligand/ion & 13160 \\
Water & 12 \\
B-factors $\left(\AA^{2}\right)$ & 655 \\
Main-chain & \\
Side-chain and water & 24.88 \\
RMS deviations & 25.91 \\
Bond lengths $(\AA)$ & \\
Bond angles $\left({ }^{\circ}\right)$ & 0.010 \\
PDB code & 1.201 \\
& $3 \mathrm{E} 04(\mathrm{doi}: 10.2210 / \mathrm{pdb} 3 \mathrm{e} 04 / \mathrm{pdb})$ \\
& \\
&
\end{tabular}

* Numbers in parentheses represent data in the highest resolution shell. features observed are hypotonia, failure to thrive, severe psychomotor retardation, seizures, facial dysmorphism and brain malformations. Interestingly, whereas homozygous FH mutations predispose to fumaric aciduria, several heterozygous $\mathrm{FH}$ mutations are known to be involved in the autosomal dominant syndrome of multiple cutaneous and uterine leiomyomata (MCUL1) (Tomlinson et al. 2002). Affected individuals develop benign smooth muscle tumours of the skin, and females develop fibroids of the uterus. When co-existing with an aggressive form of renal cell carcinoma (papillary renal type II cancer or renal collecting duct cancer) it is also known as hereditary leiomyomatosis and renal cancer (HLRCC) syndrome. In MCUL1/HLRCC germline mutations in FH are detected in the majority of the screened cases. To date, 107 variants have been described, of which 93 are thought to be pathogenic (Bayley et al. 2008). The most common types are missense mutations $(57 \%)$, followed by frameshift and nonsense mutations (27\%), as well as diverse deletions, insertions and duplications.

Here we present the crystal structure of human fumarase at $1.95 \AA$ resolution and summarize structure-activity correlation between observed mutations and clinical phenotypes.

\section{Materials and methods}

Expression, purification \& crystallization

DNA fragment encoding the fumerase domain of human $\mathrm{FH}$ (aa 44-510; GenBank entry 19743875) was subcloned into
Fig. 1 Ribbon/surface diagram of human fumarate hydratase illustrating the tetrameric assembly of class II fumarases. Molecular surface representation is used to convey the overall shape of each monomer as well as the tetrameric assembly. Each monomer has been coloured distinctively, to facilitate visualization. Two monomers are represented using semi-transparent surfaces, to highlight the fold (represented as ribbons). One of the active sites is highlighted in red, showing contribution of three distinct subunits. The figures were generated using the program ICM (www.molsoft.com)

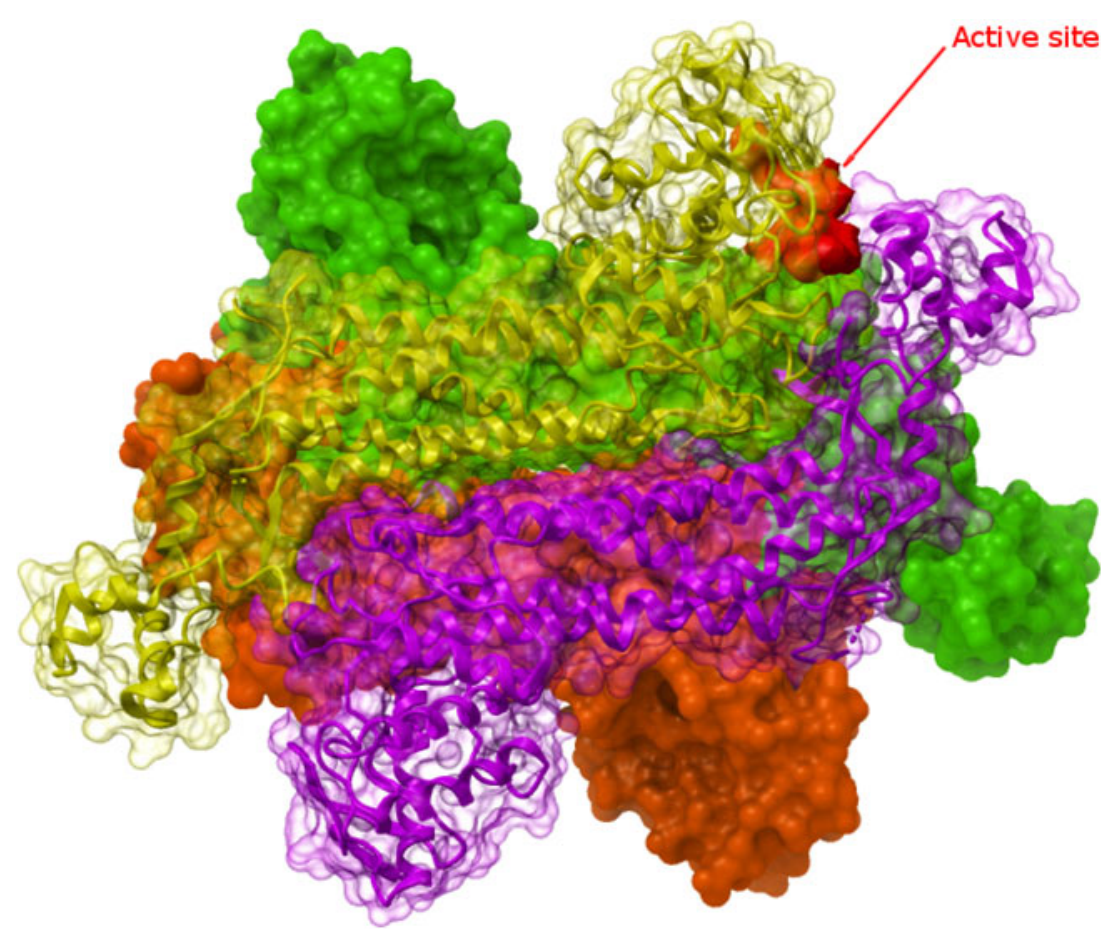


Table 2 Mutations observed in the human fumarase gene and association to disease. Abbreviations: CL: cutaneous leiomyoma; FHD: fumarate hydratase deficiency; HLRCC: hereditary leiomyomatosis and renal cell cancer; LCT: Leydig cell tumors; MCUL: multiple cutaneous and uterine leiomyomata; OMC: ovarian mucinous cystadenoma; RCC: renal cell carcinoma; STS: soft tissue sarcoma; UL: uterine leiomyomas; ULMS: uterine leiomyosarcoma

\begin{tabular}{|c|c|c|c|c|c|c|c|c|c|}
\hline \# & $\begin{array}{l}\text { Mutation } \\
\text { site }\end{array}$ & $\begin{array}{l}\text { Mutated } \\
\text { residue }\end{array}$ & $\begin{array}{l}\text { Protein } \\
\text { change }\end{array}$ & $\begin{array}{l}\text { DNA } \\
\text { change }\end{array}$ & Exon & Conservation & Localization & Reference & Phenotype \\
\hline 1 & $\operatorname{Arg} 51$ & Glu & R51E & c. $152 \mathrm{G}>\mathrm{A}$ & 2 & Conserved & Surface & (Kiuru et al. 2002) & STS \\
\hline 2 & $\operatorname{Arg} 101$ & Pro & R101P & c. $302 \mathrm{G}>\mathrm{C}$ & 3 & Semi-conserved & Surface & $\begin{array}{l}\text { (Chan et al. 2005), } \\
\text { (Heinritz et al. 2008) }\end{array}$ & HLRCC \\
\hline 3 & Asn107 & Thr & N107T & c. $320 \mathrm{~A}>\mathrm{C}$ & 3 & Conserved & Active site & $\begin{array}{l}\text { (Tomlinson et al. 2002), } \\
\text { (Alam et al. 2005a), } \\
\text { (Carvajal-Carmona et al. 2006) }\end{array}$ & MCUL, LCT \\
\hline 4 & Ala117 & Pro & A117P & c. $349 \mathrm{G}>\mathrm{C}$ & 3 & Semi-conserved & Near active site & (Tomlinson et al. 2002) & MCUL \\
\hline 5 & Leu132 & Ser & L132S & c. $395 \mathrm{~T}>\mathrm{C}$ & 4 & Semi-conserved & Surface & (Wei et al. 2006) & $\begin{array}{l}\text { HLRCC, reduced } \\
\text { FH activity }\end{array}$ \\
\hline 6 & His 135 & Arg & H135R & c. $404 \mathrm{~A}>\mathrm{G}$ & 4 & Semi-conserved & Surface & (Chuang et al. 2005) & MCUL \\
\hline 7 & Gln 142 & Lys & Q142K & c. $424 \mathrm{C}>\mathrm{A}$ & 4 & Conserved & Near active site & (Badeloe et al. 2006) & MCUL \\
\hline 8 & Ser158 & Ile & S158I & c. $473 \mathrm{G}>\mathrm{T}$ & 4 & Semi-conserved & Near active site & (Martinez-Mir et al. 2003) & MCUL \\
\hline 9 & $\operatorname{Arg} 160$ & Gly & $\mathrm{R} 160 \mathrm{G}$ & c. $478 \mathrm{~A}>\mathrm{G}$ & 4 & Conserved & Surface & (Wei et al. 2006) & $\begin{array}{l}\text { MUCL, reduced } \\
\text { FH activity }\end{array}$ \\
\hline 10 & Pro174 & Arg & P174R & c. $521 \mathrm{C}>\mathrm{G}$ & 4 & Not conserved & Surface & $\begin{array}{l}\text { (Alam et al. 2005b), } \\
\text { (Zeng et al. 2006), } \\
\text { (Pollard et al. 2005) }\end{array}$ & FHD \\
\hline 11 & His 180 & Arg & $\mathrm{H} 180 \mathrm{R}$ & c. $539 \mathrm{~A}>\mathrm{G}$ & 4 & Semi-conserved & Active site & $\begin{array}{l}\text { (Tomlinson et al. 2002), } \\
\text { (Alam et al. 2005b) }\end{array}$ & MUCL \\
\hline 12 & $\mathrm{G} \ln 185$ & $\operatorname{Arg}$ & Q185R & c. $554 \mathrm{~A}>\mathrm{G}$ & 4 & Conserved & Active site & (Tomlinson et al. 2002) & MCUL \\
\hline 13 & Ser187 & Leu & S187L & c. $560 \mathrm{C}>\mathrm{T}$ & 5 & Conserved & Active site & (Toro et al. 2003) & MCUL \\
\hline 14 & Asn188 & Ser & N188S & c. $563 \mathrm{~A}>\mathrm{G}$ & 5 & Conserved & Active site & (Toro et al. 2003) & MCUL \\
\hline 15 & Pro192 & Leu & P192L & c. $575 \mathrm{~A}>\mathrm{G}$ & 5 & Conserved & In core helice & (Chuang et al. 2005) & MCUL \\
\hline 16 & Met195 & Thr & M195T & c. $584 \mathrm{~T}>\mathrm{C}$ & 5 & Conserved & In core helice & (Toro et al. 2003) & MCUL \\
\hline 17 & His196 & Arg & H196R & c. $587 \mathrm{~A}>\mathrm{G}$ & 5 & Conserved & In core helice & $\begin{array}{l}\text { (Kiuru et al. 2002), } \\
\text { (Lehtonen et al. 2004) }\end{array}$ & RCC, ULMS \\
\hline 18 & Ile229 & Thr & I229T & c. $686 \mathrm{~T}>\mathrm{C}$ & 5 & Not conserved & Surface & (Alam et al. 2005b) & MCUL \\
\hline 19 & Lys 230 & Arg & K230R & c. $689 \mathrm{~A}>\mathrm{G}$ & 5 & Conserved & Subunit stabilization & $\begin{array}{l}\text { (Tomlinson et al. 2002), } \\
\text { (Coughlin et al. 1998), } \\
\text { (Manning et al. 2000) }\end{array}$ & FHD \\
\hline 20 & $\operatorname{Arg} 233$ & Cys & $\mathrm{R} 233 \mathrm{C}$ & c. $697 \mathrm{C}>\mathrm{T}$ & 5 & Conserved & Active site & $\begin{array}{l}\text { (Rustin et al. 1997), } \\
\text { (Chuang et al. 2005), } \\
\text { (Wei et al. 2006) }\end{array}$ & $\begin{array}{l}\text { FHD, HLRCC, } \\
\text { MCUL }\end{array}$ \\
\hline 21 & Arg233 & His & $\mathrm{R} 233 \mathrm{H}$ & c. $698 \mathrm{G}>\mathrm{A}$ & 5 & Conserved & Active site & $\begin{array}{l}\text { (Tomlinson et al. 2002), } \\
\text { (Alam et al. 2005b), (Wei et al. } \\
\text { 2006), (Chuang et al. 2005), } \\
\text { (Toro et al. 2003) }\end{array}$ & HLRCC, MCUL \\
\hline 22 & Arg233 & Leu & $\mathrm{R} 233 \mathrm{~L}$ & c. $698 \mathrm{G}>\mathrm{T}$ & 5 & Conserved & Active site & $\begin{array}{l}\text { (Chuang et al. 2005), } \\
\text { (Toro et al. 2003) }\end{array}$ & MCUL \\
\hline 23 & Ala239 & Thr & $\mathrm{A} 239 \mathrm{~T}$ & c. $715 \mathrm{G}>\mathrm{A}$ & 5 & Conserved & Near active site & (Lehtonen et al. 2004) & UL \\
\hline 24 & Ala274 & Thr & $\mathrm{A} 274 \mathrm{~T}$ & c. $820 \mathrm{G}>\mathrm{A}$ & 6 & Not conserved & Active site & (Ylisaukko-oja et al. 2006) & $\mathrm{OMC}$ \\
\hline 25 & Gly282 & Val & $\mathrm{G} 282 \mathrm{~V}$ & c. $845 \mathrm{G}>\mathrm{T}$ & 6 & Conserved & Active site & $\begin{array}{l}\text { (Tomlinson et al. 2002) } \\
\text { (Alam et al. 2005b) }\end{array}$ & MCUL \\
\hline 26 & Ala308 & Thr & $\mathrm{A} 308 \mathrm{~T}$ & c. $922 \mathrm{G}>\mathrm{A}$ & 7 & Conserved & Surface & (Coughlin et al. 1998) & FHD \\
\hline 27 & Asn310 & Tyr & $\mathrm{N} 310 \mathrm{Y}$ & c. $928 \mathrm{~A}>\mathrm{T}$ & 7 & Conserved & Surface & (Alam et al. 2005b) & MCUL \\
\hline 28 & Phe312 & Cys & $\mathrm{F} 312 \mathrm{C}$ & c. $935 \mathrm{~T}>\mathrm{G}$ & 7 & Conserved & Surface & (Coughlin et al. 1998) & FHD \\
\hline 29 & His 318 & Tyr & $\mathrm{H} 318 \mathrm{Y}$ & c. $952 \mathrm{C}>\mathrm{T}$ & 7 & Semi-conserved & In core helice & $\begin{array}{l}\text { (Toro et al. 2003), } \\
\quad \text { (Martinez-Mir et al. 2003) }\end{array}$ & HLRCC \\
\hline 30 & His 318 & Leu & H318L & c. $953 \mathrm{~A}>\mathrm{T}$ & 7 & Semi-conserved & In core helice & (Deschauer et al. 2006) & FHD \\
\hline 31 & Val322 & Asp & V322D & c. $964 \mathrm{~T}>\mathrm{A}$ & 7 & Conserved & $\begin{array}{l}\text { In core helice (interaction } \\
\text { with } 1 \text { other monomer) }\end{array}$ & (Toro et al. 2003) & MCUL \\
\hline 32 & Thr330 & Pro & T330P & c. $988 \mathrm{~A}>\mathrm{C}$ & 7 & Semi-conserved & $\begin{array}{l}\text { In core helice (interaction } \\
\text { with } 1 \text { other monomer) }\end{array}$ & (Chuang et al. 2005) & MCUL \\
\hline 33 & Cys333 & Tyr & $\mathrm{C} 333 \mathrm{Y}$ & c. $998 \mathrm{G}>\mathrm{A}$ & 7 & Semi-conserved & $\begin{array}{l}\text { In core helice (interaction } \\
\text { with } 1 \text { other monomer) }\end{array}$ & & MCUL \\
\hline 34 & Ser334 & Arg & S334R & c. $1002 \mathrm{~T}>\mathrm{G}$ & 7 & Conserved & $\begin{array}{l}\text { In core helice (interaction } \\
\text { with } 1 \text { other monomer) }\end{array}$ & (Badeloe et al. 2006) & $\mathrm{CL}$ \\
\hline 35 & Leu335 & Pro & L335P & c. $1004 \mathrm{~T}>\mathrm{C}$ & 7 & Conserved & In core helice & (Toro et al. 2003) & MCUL \\
\hline
\end{tabular}


Table 2 (continued)

\begin{tabular}{|c|c|c|c|c|c|c|c|c|c|}
\hline$\#$ & $\begin{array}{l}\text { Mutation } \\
\text { site }\end{array}$ & $\begin{array}{l}\text { Mutated } \\
\text { residue }\end{array}$ & $\begin{array}{l}\text { Protein } \\
\text { change }\end{array}$ & $\begin{array}{l}\text { DNA } \\
\text { change }\end{array}$ & Exon & Conservation & Localization & Reference & Phenotype \\
\hline 36 & Asn 340 & Lys & $\mathrm{N} 340 \mathrm{~K}$ & c. $1020 \mathrm{~T}>\mathrm{A}$ & 7 & Semi-conserved & In core helice & $\begin{array}{l}\text { (Toro et al. 2003), } \\
\text { (Wei et al. 2006) }\end{array}$ & MCUL \\
\hline 37 & Glu355 & Lys & E355K & c. $1063 \mathrm{G}>\mathrm{A}$ & 7 & Conserved & Subunit stabilization & (Alam et al. 2005b) & MCUL \\
\hline 38 & Asn361 & Lys & $\mathrm{N} 361 \mathrm{~K}$ & c. $1083 \mathrm{~T}>\mathrm{A}$ & 7 & Conserved & Active site & (Alam et al. 2005b) & HLRCC-CDC \\
\hline 39 & Glu362 & Gln & E362Q & c. $1084 \mathrm{G}>\mathrm{C}$ & 7 & Conserved & Active site & (Bourgeron et al. 1994) & FHD \\
\hline 40 & Ser365 & Gly & S365G & c. $1093 \mathrm{G}>\mathrm{A}$ & 7 & Conserved & Active site & $\begin{array}{l}\text { (Toro et al. 2003), } \\
\text { (Wei et al. 2006) }\end{array}$ & MCUL \\
\hline 41 & Ser366 & Asn & $\mathrm{S} 366 \mathrm{~N}$ & c. $1097 \mathrm{G}>\mathrm{A}$ & 7 & Conserved & Active site (but out) & $\begin{array}{l}\text { (Toro et al. 2003), } \\
\text { (Alam et al. 2005b) }\end{array}$ & MCUL \\
\hline 42 & Met368 & Thr & M368T & c. $1103 \mathrm{~T}>\mathrm{C}$ & 7 & Conserved & Active site & (Badeloe et al. 2006) & MCUL \\
\hline 43 & Pro369 & Ser & P369S & c. $1105 \mathrm{C}>\mathrm{T}$ & 7 & Conserved & Active site (but out) & (Maradin et al. 2006) & FHD \\
\hline 44 & Asn 373 & Ser & N373S & c. $1118 \mathrm{~A}>\mathrm{G}$ & 8 & Conserved & Active site & (Lehtonen et al. 2004) & $\begin{array}{l}\text { HLRCC/clear } \\
\text { cell RCC }\end{array}$ \\
\hline 45 & $\mathrm{G} \ln 376$ & Pro & Q376P & c. $1127 \mathrm{~A}>\mathrm{C}$ & 8 & Conserved & $\begin{array}{l}\text { In core helice (interaction } \\
\text { with } 1 \text { other monomer) }\end{array}$ & $\begin{array}{l}\text { (Zeman et al. 2000), } \\
\text { (Remes et al. 2004), } \\
\text { (Phillips et al. 2006) }\end{array}$ & FHD \\
\hline 46 & Ala385 & Asp & A385D & c. $1154 \mathrm{C}>\mathrm{A}$ & 8 & Not conserved & $\begin{array}{l}\text { In core helice (interaction } \\
\text { with } 2 \text { other monomers) }\end{array}$ & (Wei et al. 2006) & MCUL \\
\hline 47 & Val394 & Leu & V394L & c. $1180 \mathrm{G}>\mathrm{C}$ & 8 & Not conserved & In core helice & (Martinez-Mir et al. 2003) & MCUL \\
\hline 48 & Gly397 & Arg & G397R & c. $1189 \mathrm{G}>\mathrm{A}$ & 8 & Semi- conserved & In core helice & (Alam et al. 2005b) & MCUL \\
\hline 49 & His402 & Cys & $\mathrm{H} 402 \mathrm{C}$ & c. $1207 \mathrm{C}>\mathrm{T}$ & 8 & Conserved & $\begin{array}{l}\text { In core helice turn (interaction } \\
\text { with } 2 \text { other monomers) }\end{array}$ & (Phillips et al. 2006) & FHD \\
\hline 50 & Ser419 & Pro & S419P & c. $1255 \mathrm{~T}>\mathrm{C}$ & 9 & Conserved & In core helice & (Wei et al. 2006) & HLRCC \\
\hline 51 & Asp425 & Val & D425V & c. $1274 \mathrm{~A}>\mathrm{T}$ & 9 & Conserved & $\begin{array}{l}\text { In core helice (interaction } \\
\text { with } 1 \text { other monomer) }\end{array}$ & (Coughlin et al. 1998) & FHD \\
\hline 52 & $\mathrm{G} \ln 439$ & Pro & Q439P & c. $1316 \mathrm{~A}>\mathrm{C}$ & 9 & Not conserved & Surface & (Wei et al. 2006) & HLRCC \\
\hline 53 & Met454 & Ile & M454I & c. $1362 \mathrm{G}>\mathrm{A}$ & 9 & Conserved & Subunit interaction & (Carvajal-Carmona et al. 2006) & LCT \\
\hline 54 & Tyr465 & Cys & $\mathrm{Y} 465 \mathrm{C}$ & c. $1394 \mathrm{~A}>\mathrm{G}$ & 10 & Semi- conserved & Surface & (Toro et al. 2003) & MCUL \\
\hline 55 & Leu507 & Pro & L507P & c. $1520 \mathrm{~T}>\mathrm{C}$ & 10 & Semi- conserved & $\begin{array}{l}\text { Surface near opening } \\
\text { active site }\end{array}$ & (Alam et al. 2005b) & MCUL \\
\hline
\end{tabular}

Fig. 2 Clustering of human fumarase missense mutations observed in FHD, MCUL1 and HLRCC. The active site is highlighted in cyan. Positions of amino acid mutations are indicated as small spheres and numbered according to Table 2 . The positions around the active site are indicated in red, mutations affecting interor intrasubunit interactions are indicated in dark yellow. For clarity, one monomeric subunit is omitted

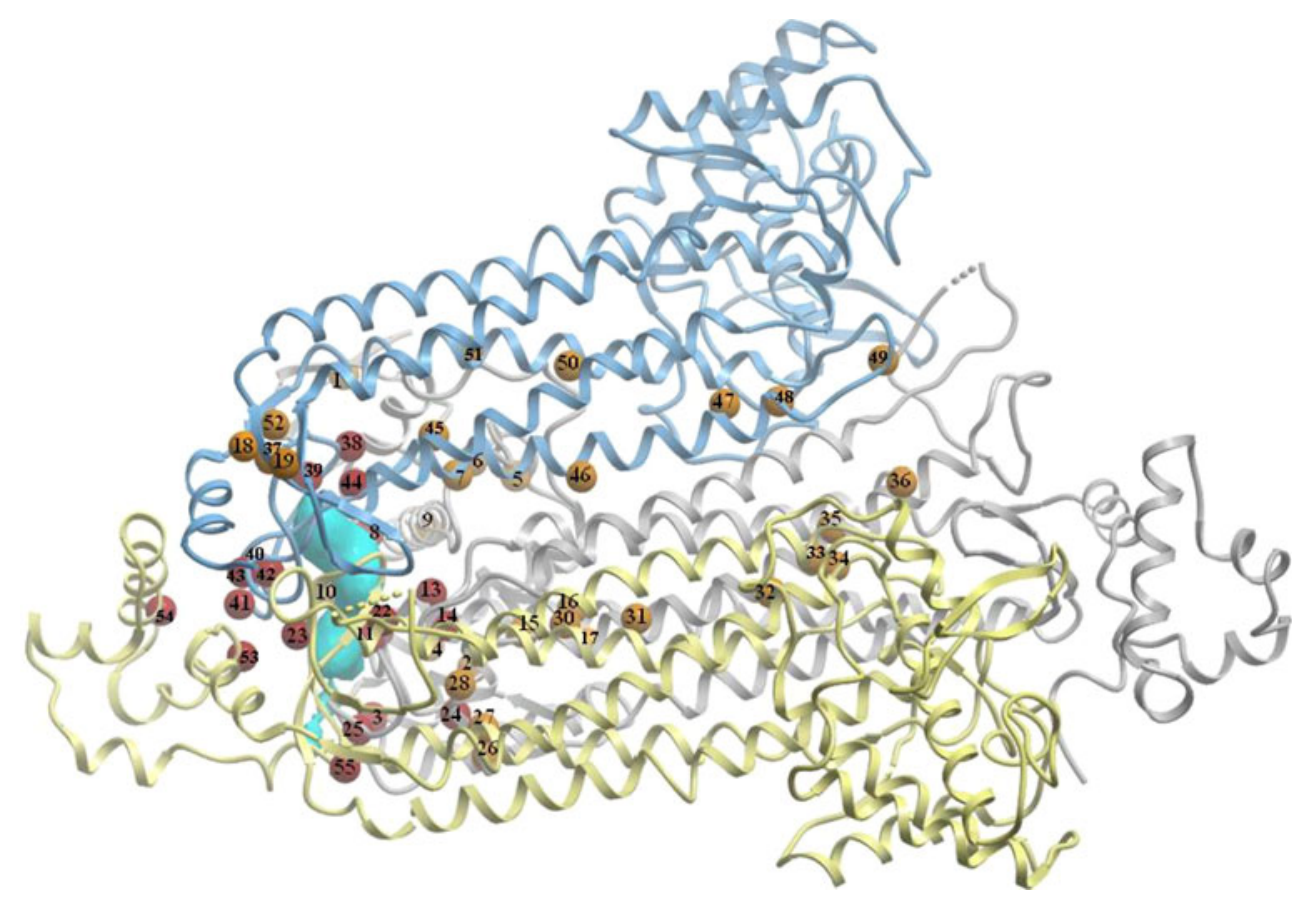


pNIC28-Bsa4 vector incorporating an N-terminal His $_{6}$-tag. The plasmid was transformed into BL21(DE3)-pRARE, cultured in Terrific Broth at $37^{\circ} \mathrm{C}$, and induced with $0.1 \mathrm{mM}$ IPTG overnight at $18^{\circ} \mathrm{C}$. Cells were homogenized in lysis buffer (50 mM K-phosphate pH 7.5, $500 \mathrm{mM} \mathrm{NaCl,} 1 \mathrm{mM}$ TCEP), centrifuged to remove cell debris, and the supernatant was purified by Nickel affinity (HisTrap Crude FF) and size exclusion (HiLoad 16/60 Superdex S200) chromatography. Purified protein was concentrated to $12.6 \mathrm{mg} / \mathrm{ml}$ and stored in $10 \mathrm{mM}$ HEPES pH 7.5, $150 \mathrm{mM} \mathrm{NaCl}, 5 \%$ (w/v) glycerol and $0.5 \mathrm{mM}$ TCEP at $-80^{\circ} \mathrm{C}$. Crystals were grown by vapour diffusion at $20^{\circ} \mathrm{C}$ in sitting drops mixing $150 \mathrm{nl}$ protein and $150 \mathrm{nl}$ reservoir solution containing 20\% (w/v) PEG 3350, $0.2 \mathrm{M}$ sodium acetate, $10 \%(\mathrm{w} / \mathrm{v})$ ethylene glycol and $100 \mathrm{mM}$ Bis-Tris propane $\mathrm{pH}$ 7.5. Crystals were cryoprotected in mother liquor containing $25 \%(\mathrm{w} / \mathrm{v})$ glycerol and flash-frozen in liquid nitrogen.

\section{Data collection \& structure determination}

Diffraction data to maximum resolution of $1.95 \AA$ were collected on beamline X10A at the Swiss Light Source, and processed using the CCP4 Program suite (CCP4, 1994). FH crystallized in the trigonal space group $\mathrm{P}_{2} 21(a=180.5 \AA$, $b=180.5 \AA, c=114.6 \AA, \alpha=90^{\circ}, \beta=90^{\circ}, \gamma=120^{\circ}$ ) with four molecules in the asymmetric unit. The structure of FH was solved by molecular replacement with PHASER (McCoy et al. 2005), using the yeast fumerase structure as search model (PDB code 1YFM). Initial automated model building was performed with ARP/wARP (Perrakis et al. 1999). This is followed by cycles of iterative manual model building using COOT (Emsley \& Cowtan 2004) and restrained refinement using REFMAC5 with TLS parameters (Murshudov et al. 1997). The final structure was deposited in the Protein Data Bank (www.rcsb.org) under accession code 3E04 (Table 1).

\section{Results and discussion}

Fumarases are divided into two distinct groups. Class I fumarases are iron-dependent iron-sulfur cluster containing, dimeric enzymes, whereas the class II enzymes, including human and other eukaryotic fumarases, are homotetrameric enzymes with a molecular mass of about $200 \mathrm{kDa}$. Class II fumarases are evolutionarily highly conserved enzymes, e.g. the pairwise identity between $E$. coli and human fumarase is about $60 \%$. Every monomer exhibits a typical tridomain structure, with a central domain involved in subunit interaction, thus forming a typical bundle comprised of 20 $\alpha$-helices (Fig. 1A). Previous crystallographic analyses have revealed two distinct sites (A and $\mathrm{B}$ ) in $E$. coli fumarase that can bind carboxylic acids. Site $\mathrm{A}$ is formed from three different monomer chains and likely to be the catalytic site, whereas site B is thought to allosterically regulate activity (Rose and Weaver 2004).

A previous study correlated 27 distinct missense mutations to the E. coli fumarase structure (Alam 2005b), since then the list of mutations has doubled. To this end, 55 missense mutations in the human fumarase gene are now described. Here we correlate this updated list of mutations to fumarase deficiency, MCUL1 and HLRCC syndrome (Table 2) by using the human fumarase structure. Although not all of these novel mutations have been biochemically characterized, previous results suggest that $\mathrm{FH}$ activity is related to HLRCC (Alam 2005a), although other environmental or genetic factors likely play a role in the etiology of the disease. The clustering of mutational "hotspots" suggests enzyme activity relationships to phenotypic appearances. Figure 2 illustrates the clustering of $\mathrm{FH}$ mutations observed in FHD, MCUL1 and HLRCC. The large majority of mutations are located at evolutionarily highly conserved positions (Table 2) indicating that these mutations likely affect stability and/or activity of the enzyme. Two major clusters of mutations are observed; one is likely to affect structural integrity of the enzyme by interrupting inter or intrasubunit interactions (indicated in yellow in Fig. 2), whereas the other mutations are located around the active site and likely directly affect activity.

Acknowledgments Help in data collection at SLS (Swiss Light Source, Villigen, CH) by Frank von Delft, Annette Roos and Panagis Filippakopoulos is gratefully acknowledged. The Structural Genomics Consortium is a registered charity (Number 1097737) funded by the Canadian Institutes for Health Research, the Canadian Foundation for Innovation, Genome Canada through the Ontario Genomic institute, GlaxoSmithKline, Karolinska Institutet, the Knut and Alice Wallenberg Innovation, Merck and Co., Inc., the Novartis Research Foundation, the Swedish Agency for Innovation Systems, the Swedish Foundation for Strategic Research and the Wellcome Trust. The study was supported by the NIHR Oxford Biomedical Research Unit.

Open Access This article is distributed under the terms of the Creative Commons Attribution Noncommercial License which permits any noncommercial use, distribution, and reproduction in any medium, provided the original author(s) and source are credited.

\section{References}

Alam NA, Olpin S, Leigh IM (2005a) Fumaratehydratase mutations and predisposition to cutaneous leiomyomas, uterine leiomyomas and renal cancer. Br J Dermatol 153:11-17

Alam NA, Olpin S, Rowan A, Kelsell D, Leigh IM, Tomlinson IP, Weaver T (2005b) Missense mutations in fumaratehydratase in multiple cutaneous and uterine leiomyomatosis and renal cell cancer. J Mol Diagn 7:437-443

Badeloe S, van Geel M, van Steensel MA, Bastida J, Ferrando J, Steijlen PM, Frank J, Poblete-Gutierrez P (2006) Diffuse and segmental variants of cutaneous leiomyomatosis: novel mutations in the fumaratehydratase gene and review of the literature. Exp Dermatol 15:735-741 
Bayley JP, Launonen V, Tomlinson IP (2008) The FH mutation database: an online database of fumaratehydratase mutations involved in the MCUL (HLRCC) tumor syndrome and congenital fumarase deficiency. BMC Med Genet 9:20

Bourgeron T, Chretien D, Poggi-Bach J, Doonan S, Rabier D, Letouze P, Munnich A, Rotig A, Landrieu P, Rustin P (1994) Mutation of the fumarase gene in two siblings with progressive encephalopathy and fumarase deficiency. J Clin Invest 93:2514-2518

Carvajal-Carmona LG, Alam NA, Pollard PJ, Jones AM, Barclay E, Wortham N, Pignatelli M, Freeman A, Pomplun S, Ellis I, Poulsom R, El-Bahrawy MA, Berney DM, Tomlinson IP (2006) Adult leydig cell tumors of the testis caused by germlinefumaratehydratase mutations. J Clin Endocrinol Metab 91:3071-3075

CCP4 (1994) The CCP4 suite: programs for protein crystallography. Acta Crystallogr D Biol Crystallogr 50:760-763

Chan I, Wong T, Martinez-Mir A, Christiano AM, McGrath JA (2005) Familial multiple cutaneous and uterine leiomyomas associated with papillary renal cell cancer. Clin Exp Dermatol 30:75-78

Chuang GS, Martinez-Mir A, Geyer A, Engler DE, Glaser B, Cserhalmi-Friedman PB, Gordon D, Horev L, Lukash B, Herman E, Cid MP, Brenner S, Landau M, Sprecher E, Garcia Muret MP, Christiano AM, Zlotogorski A (2005) Germlinefumaratehydratase mutations and evidence for a founder mutation underlying multiple cutaneous and uterine leiomyomata. J Am Acad Dermatol 52:410-416

Coughlin EM, Christensen E, Kunz PL, Krishnamoorthy KS, Walker V, Dennis NR, Chalmers RA, Elpeleg ON, Whelan D, Pollitt RJ, Ramesh V, Mandell R, Shih VE (1998) Molecular analysis and prenatal diagnosis of human fumarase deficiency. Mol Genet Metab 63:254-262

Deschauer M, Gizatullina Z, Schulze A, Pritsch M, Knoppel C, Knape M, Zierz S, Gellerich FN (2006) Molecular and biochemical investigations in fumarase deficiency. Mol Genet Metab 88:146-152

Emsley P, Cowtan K (2004) Coot: model-building tools for molecular graphics. Acta Crystallogr D Biol Crystallogr 60:2126-2132

Heinritz W, Paasch U, Sticherling M, Wittekind C, Simon JC, Froster UG, Renner R (2008) Evidence for a founder effect of the germlinefumaratehydratase gene mutation R58P causing hereditary leiomyomatosis and renal cell cancer (HLRCC). Ann Hum Genet 72:35-40

Kerrigan JF, Aleck KA, Tarby TJ, Bird CR, Heidenreich RA (2000) Fumaric aciduria: clinical and imaging features. Ann Neurol 47:583-588

King A, Selak MA, Gottlieb E (2006) Succinate dehydrogenase and fumaratehydratase: linking mitochondrial dysfunction and cancer. Oncogene 25:4675-4682

Kiuru M, Lehtonen R, Arola J, Salovaara R, Jarvinen H, Aittomaki K, Sjoberg J, Visakorpi T, Knuutila S, Isola J, Delahunt B, Herva R, Launonen V, Karhu A, Aaltonen LA (2002) Few FH mutations in sporadic counterparts of tumor types observed in hereditary leiomyomatosis and renal cell cancer families. Cancer Res 62:4554-4557

Lehtonen R, Kiuru M, Vanharanta S, Sjoberg J, Aaltonen LM, Aittomaki K, Arola J, Butzow R, Eng C, Husgafvel-Pursiainen $\mathrm{K}$, Isola J, Jarvinen H, Koivisto P, Mecklin JP, Peltomaki P, Salovaara R, Wasenius VM, Karhu A, Launonen V, Nupponen NN, Aaltonen LA (2004) Biallelic inactivation of fumaratehydratase $(\mathrm{FH})$ occurs in nonsyndromic uterine leiomyomas but is rare in other tumors. Am J Pathol 164:17-22

McCoy AJ, Grosse-Kunstleve RW, Storoni LC, Read RJ (2005) Likelihood-enhanced fast translation functions. Acta Crystallogr D Biol Crystallogr 61:458-464

Manning NJ, Olpin SE, Pollitt RJ, Downing M, Heeley AF, Young ID (2000) Fumaratehydratase deficiency: increased fumaric acid in amniotic fluid of two affected pregnancies. J Inherit Metab Dis 23:757-759

Maradin M, Fumic K, Hansikova H, Tesarova M, Wenchich L, Dorner S, Sarnavka V, Zeman J, Baric I (2006) Fumaricaciduria: mild phenotype in a 8-year-old girl with novel mutations. J Inherit Metab Dis 29:683

Martinez-Mir A, Glaser B, Chuang GS, Horev L, Waldman A, Engler DE, Gordon D, Spelman LJ, Hatzibougias I, Green J, Christiano AM, Zlotogorski A (2003) Germlinefumaratehydratase mutations in families with multiple cutaneous and uterine leiomyomata. J Invest Dermatol 121:741-744

Murshudov GN, Vagin AA, Dodson EJ (1997) Refinement of macromolecular structures by the maximum-likelihood method. Acta Crystallogr D Biol Crystallogr 53:240-255

Perrakis A, Morris R, Lamzin VS (1999) Automated protein model building combined with iterative structure refinement. Nat Struct Biol 6:458-463

Phillips TM, Gibson JB, Ellison DA (2006) Fumaratehydratase deficiency in monozygotic twins. Pediatr Neurol 35:150-153

Pollard PJ, Briere JJ, Alam NA, Barwell J, Barclay E, Wortham NC, Hunt T, Mitchell M, Olpin S, Moat SJ, Hargreaves IP, Heales SJ, Chung YL, Griffiths JR, Dalgleish A, McGrath JA, Gleeson MJ, Hodgson SV, Poulsom R, Rustin P, Tomlinson IP (2005) Accumulation of Krebs cycle intermediates and over-expression of HIF1alpha in tumours which result from germline FH and SDH mutations. Hum Mol Genet 14:2231-2239

Ratcliffe PJ (2007) Fumaratehydratase deficiency and cancer: activation of hypoxia signaling? Cancer Cell 11:303-305

Remes AM, Filppula SA, Rantala H, Leisti J, Ruokonen A, Sharma S, Juffer AH, Hiltunen JK (2004) A novel mutation of the fumarase gene in a family with autosomal recessive fumarase deficiency. J Mol Med 82:550-554

Rose IA, Weaver TM (2004) The role of the allosteric B site in the fumarase reaction. Proc Natl Acad Sci U S A 101:3393-3397

Rustin P, Bourgeron T, Parfait B, Chretien D, Munnich A, Rotig A (1997) Inborn errors of the Krebs cycle: a group of unusual mitochondrial diseases in human. Biochim Biophys Acta 1361:185-197

Tomlinson IP, Alam NA, Rowan AJ, Barclay E, Jaeger EE, Kelsell D, Leigh I, Gorman P, Lamlum H, Rahman S, Roylance RR, Olpin S, Bevan S, Barker K, Hearle N, Houlston RS, Kiuru M, Lehtonen R, Karhu A, Vilkki S, Laiho P, Eklund C, Vierimaa O, Aittomaki K, Hietala M, Sistonen P, Paetau A, Salovaara R, Herva R, Launonen V, Aaltonen LA (2002) Germline mutations in FH predispose to dominantly inherited uterine fibroids, skin leiomyomata and papillary renal cell cancer. Nat Genet 30:406-410

Toro JR, Nickerson ML, Wei MH, Warren MB, Glenn GM, Turner ML, Stewart L, Duray P, Tourre O, Sharma N, Choyke P, Stratton P, Merino M, Walther MM, Linehan WM, Schmidt LS, Zbar B (2003) Mutations in the fumaratehydratase gene cause hereditary leiomyomatosis and renal cell cancer in families in North America. Am J Hum Genet 73:95-106

Wei MH, Toure O, Glenn GM, Pithukpakorn M, Neckers L, Stolle C, Choyke P, Grubb R, Middelton L, Turner ML, Walther MM, Merino MJ, Zbar B, Linehan WM, Toro JR (2006) Novel mutations in $\mathrm{FH}$ and expansion of the spectrum of phenotypes expressed in families with hereditary leiomyomatosis and renal cell cancer. J Med Genet 43:18-27

Ylisaukko-oja SK, Cybulski C, Lehtonen R, Kiuru M, Matyjasik J, Szymanska A, Szymanska-Pasternak J, Dyrskjot L, Butzow R, Orntoft TF, Launonen V, Lubinski J, Aaltonen LA (2006) Germlinefumaratehydratase mutations in patients with ovarian mucinous cystadenoma. Eur J Hum Genet 14:880-883

Zeman J, Krijt J, Stratilova L, Hansikova H, Wenchich L, Kmoch S, Chrastina P, Houstek J (2000) Abnormalities in succinylpurines in fumarase deficiency: possible role in pathogenesis of CNS impairment. J Inherit Metab Dis 23:371-374

Zeng WQ, Gao H, Brueton L, Hutchin T, Gray G, Chakrapani A, Olpin S, Shih VE (2006) Fumarase deficiency caused by homozygous P131R mutation and paternal partial isodisomy of chromosome 1. Am J Med Genet A 140:1004-1009 